\title{
Association between maternal diabetes and congenital heart diseases in fetuses amongst UAE residents between 2015 and 20I7: a retrospective cohort study
}

\begin{abstract}
Objective: Determine the prevalence of congenital heart diseases in fetuses amongst mothers who are diabetic and to determine the relationship between maternal diabetes and types of congenital heart diseases in fetuses.

Methods: A proposal has been sent to and approved by Mohammad Bin Rashid University's Institutional Review Board in 2018 before the collection and analysis of the data. The approval code is: MBRU-IRB-SRP2018-046.

A descriptional retrospective cohort study was designed to analyze secondary data of forty-five, randomly selected patient files from Al Jalila Children's Hospital from 2015 to 2017. The files selected were files of diabetic mothers and the variables included: type of diabetes, type of congenital heart disease and gestational age of fetuses when echocardiography was performed.

Results: The results of this retrospective cohort study have shown that maternal diabetes may affect the development of fetal hearts and may lead to congenital heart diseases. Also, maternal diabetes type 1 may cause more congenital heart disease in infants of diabetic mothers compared to mothers who were diagnosed with diabetes type 2.

Conclusion: Since maternal diabetes was suggested to cause congenital heart diseases, it is important that mothers know about the possible effects of diabetes on the children. Diet and management must be maintained throughout the pregnancy and diabetic mothers must have their fetuses screened throughout their pregnancies.
\end{abstract}

Keywords: congenital heart diseases, diabetic mothers, echocardiograph, pregnancy
Volume II Issue 6 - 2018

\author{
Mahmoud Alsoufi,' Maha Ali Qutami \\ AlSuwaidi \\ 'Al Jalila Children's Hospital, United Arab Emirates \\ ${ }^{2}$ Mohammed Bin Rashid University of Medicine and Health \\ Sciences, College of Medicine, United Arab Emirates
}

Correspondence: Mahmoud Alsoufi, Al Jalila Children's Hospital, United Arab Emirates, Email dr.m.sowfi@hotmail.com

Received: December 03, 2018 | Published: December |4, 2018

\section{Objectives}

\section{What is already known about this subject?}

a. Some studies suggested that there was an association between diabetic mothers and congenital heart diseases in their children

b. On the other hand, some studies suggested that there is no association between diabetic mothers and congenital heart diseases in infants of diabetic mothers.

\section{What does this study add?}

a. This study was conducted in the UAE, specifically Al Jalila Children's Hospital

How might this impact on public clinical (delete as appropriate) practice?

a. A diabetic mother is advised to get an echocardiograph done throughout her pregnancy, by doing so, doctors may be able to detect an anomaly and reverse it- if the anomaly is reversible.

b. The results of this study aim to encourage diabetic mothers to control their glucose and take their medications if they decide to conceive.

\section{Introduction}

According to the International Diabetes Federation, over $1,180,000(15.6 \%)$ adult cases in the United Arab Emirates in 2017 were diabetic. ${ }^{1}$ Diabetes has been a medical issue locally and globally, due to its increasing prevalence and recorded complications. Diabetes, a preventable and manageable metabolic disease that is classified as hereditary or caused by an unhealthy lifestyle has been a regional and an international health concern. According to research and evidence-based medicine, diabetes has almost in all cases led to cardiovascular disorders. ${ }^{2}$ Recently, a question has been frequently raised about the risk factors of maternal diabetes and its effects on their foetuses. ${ }^{3}$ Many studies have reported a number of various systemic foetal abnormalities in mothers who were hyperglycaemic. ${ }^{4}$ Foetal congenital heart diseases may lead to serious complications and may occasionally be fatal. Foetal congenital heart diseases are divided into two main categories: cyanotic and acyanotic anomalies. Generally, foetuses with acyanotic cardiac malformations present with normal biological features. Moreover, a foetus would look normally red due to a left-to-right shunt which allows the blood in a heart to mix. Some of the acyanotic heart malformations include: atrial septal defect (ASD), ventricular septal defect (VSD), atrioventricular defect (ACSD), patent ductus arteriosus (PDA) and pulmonary stenosis 
(PS). ${ }^{5}$ On the other hand, cyanotic anomalies leave the foetus cyanotic and some of the examples include: Ebstein anomaly, Tetralogy of Fallot, pulmonary atresia, transposition of great vessels and truncus arteriosus. ${ }^{6}$ The mentioned examples of congenital heart diseases maybe be reversed by drugs or surgery and some cases progress to serious complications. The most common complications of cardiac anomalies include: serious irregular heartbeats (arrythmias), pulmonary hypertension, heart failure and inflammation of the inner layer of the heart (endocarditis). ${ }^{7}$

\section{Methods}

\section{Study design}

Observational retrospective cohort study analysing 45 randomly selected secondary data of case files from Al Jalila Children's Hospital in a timeframe from 2015 to 2017.

\section{Study setting}

Al Jalila Children's Hospital (AJCH) is a tertiary paediatric hospital; the departments are sub specialized into numerous units. The cardiac unit in Al Jalila Children's Hospital ensures that a foetus' heart develops healthily; therefore, the cardiac department had created policies to support foetal heart growth. One of the polices proposes that every diabetic mother who presents for consultation must be educated on the effects of diabetes on her child's heart and is then scheduled for an echocardiograph appointment. According to the case files collected and analysed, mothers had their echocardiographs done between the gestational age of 19 weeks and 38 weeks, with a mean gestational age of 28.89 weeks (Table 1). A random collection of 45 file cases of diabetic mothers were retrospectively analysed.

\section{Study variables}

Study variables include: type of diabetes, treatment (medication or diet), gestational age when echocardiograph was performed and type of congenital heart anomaly. The two main types of diabetes that were recorded were either diabetes type 1 (DT1) or diabetes type 2 (DT2). Throughout a pregnancy, a mother's foetus would be screened for any heart anomalies at any gestational week after the child has passed the embryonic stage. In case of an identified anomaly, the findings would be recorded in the patient's file and the patient would be then referred to specialist, consultant and occasionally a therapist.

\section{Biases}

Although this retrospective cohort study was designed in a way to rule out as many biases as we could, the study may have been introduced to some biases along the way. For instance, history taking by the physicians who consulted the mothers may have been superficial or brief. Moreover, recall bias may be introduced in history taking, some mothers may not remember if they have a family history or congenital heart diseases. Furthermore, whether or not the mother adhered to the treatment plan is unclear.

\section{Results}

\section{Participants}

Out of the randomly selected 45 diabetic mothers, 11(24.44\%) had foetal heart anomalies recorded in their files (Figure 1). There were 7 main abnormal findings. Furthermore, 23 out of 45 mothers had diabetes type 2 and 22 out of those 45 mothers had diabetes type 1 (Figure 2).

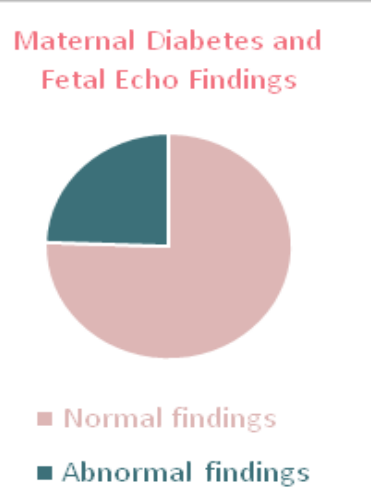

Figure I Normal versus Abnormal foetal echocardiograph findings.

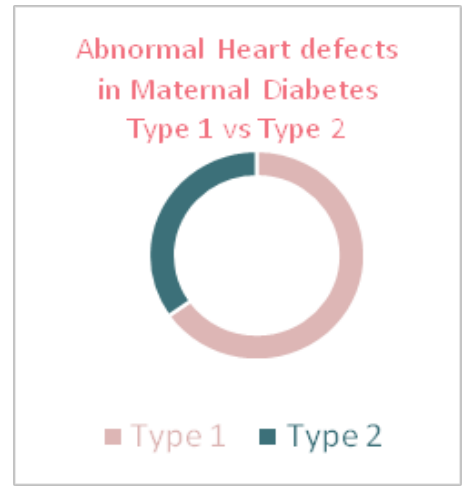

Figure 2 The effects of maternal diabetes type I versus the effects of maternal diabetes type 2 on abnormal findings.

\section{Descriptive data}

The anomalies found in the 11 affected mothers included: small heart $(11 \%)$, ventricular septal defect $(23 \%)$, mild turbulence $(11 \%)$, abnormal ventricular septal defect and dilated right heart $(11 \%)$, ventricular hypertrophy (11\%), cardiomegaly with dysplastic pulmonary valve and a dilated right heart $(11 \%)$, Ebstein anomaly $(11 \%)$ and truncus anomaly (11\%) (Figure 3$)$. Out of the 23 diabetic mothers with diabetes type 2, 4 participants had abnormal foetal findings $(0.17 \%$ out of the population of diabetes type 2 , or $0.088 \%$ out of the whole population, which was 45 mothers). Moreover, out of the 22 diabetic mothers with diabetes type 1,7 participants had abnormal findings $(0.31 \%$ out of the population of diabetes type 1 , or $0.15 \%$ out of the entire population of 45 mothers).

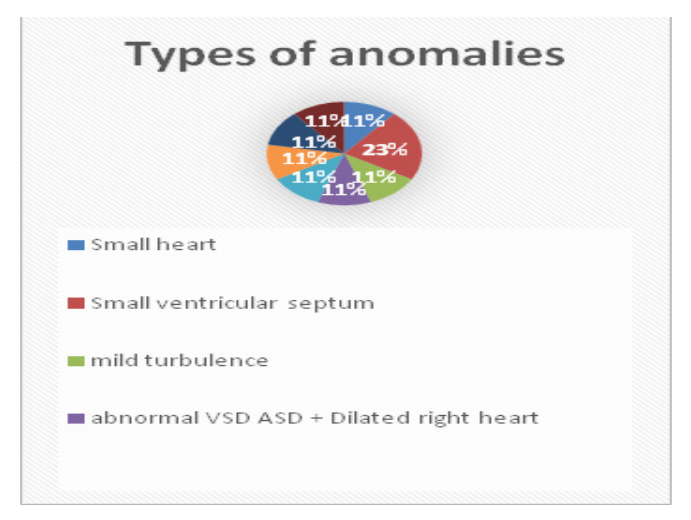

Figure 3 The 7 types of anomalies found in foetal echocardiography. 
Table I Gestational age table with mean

\begin{tabular}{lll}
\hline \multicolumn{2}{l}{ Statistics } \\
\hline \multicolumn{3}{l}{ Gestational age } \\
\hline $\mathrm{N}$ & Valid & 45 \\
& Missing & \\
Mean & & 28.89 \\
\hline
\end{tabular}

\begin{tabular}{lllll}
\multicolumn{2}{l}{ Gestational age } & & \multicolumn{2}{l}{} \\
\cline { 2 - 3 } & Frequency & Percent & $\begin{array}{l}\text { Valid } \\
\text { percent }\end{array}$ & $\begin{array}{l}\text { Cumulative } \\
\text { percent }\end{array}$ \\
\hline Valid 19 & 1 & 2.2 & 2.2 & 2.2 \\
21 & 4 & 8.9 & 8.9 & 11.1 \\
22 & 2 & 4.4 & 4.4 & 15.6 \\
24 & 2 & 4.4 & 4.4 & 20 \\
25 & 3 & 6.7 & 6.7 & 26.7 \\
26 & 4 & 8.9 & 8.9 & 35.6 \\
27 & 1 & 2.2 & 2.2 & 37.8 \\
28 & 5 & 11.1 & 11.1 & 48.9 \\
29 & 1 & 2.2 & 2.2 & 51.1 \\
30 & 3 & 6.7 & 6.7 & 57.8 \\
31 & 3 & 6.7 & 6.7 & 64.4 \\
32 & 4 & 8.9 & 8.9 & 73.3 \\
33 & 2 & 4.4 & 4.4 & 77.8 \\
34 & 4 & 8.9 & 8.9 & 86.7 \\
35 & 3 & 6.7 & 6.7 & 93.3 \\
37 & 2 & 4.4 & 4.4 & 97.8 \\
38 & 1 & 2.2 & 2.2 & 100 \\
Total & 45 & 100 & 100 & \\
\hline & & & & \\
\hline
\end{tabular}

\section{Discussion}

\section{Summary of major findings}

This is the first study performed, analysed and recorded from Al Jalila Children's Hospital, Dubai, UAE between 2015 and 2017. After collecting and analysing the data, we have concluded that 11 $(24.44 \%)$ in every 45 reported maternal diabetic cases will have abnormal echocardiograph findings. Moreover, the most common abnormal finding was ventricular septal defect. In relation with the type of diabetes, diabetes type 1 showed a higher risk of developing a foetal cardiac anomaly.

\section{Comparision with previous studies}

In a previous study, it was done in the 2000 s by two paediatric specialists: H Narchi and N Kulaylat, their study suggested that cardiac anomalies are found commonly in infants of diabetic mothers. Even though the sample size was not mentioned, their study had shown that the risk of presenting with congenital heart diseases in foetuses of diabetic mothers was between $2.5 \%$ and $12 \%$. Moreover, some of the anomalies reported by the consultants included: general structural malformations, failure of the cardiovascular system to adapt to the extrauterine life, cardiac hypertrophy and cardiomegaly. They also mentioned some managements and measures taken when a foetus is reported to have a cardiac anomaly such as drugs and procedures. ${ }^{8}$ In study done in the University of California by Dr. Lisa $\mathrm{K}$ Hornberger, the study suggested that there is a positive correlation between maternal diabetes and congenital cardiac diseases in their infants. The study suggested that the most common abnormal finding and complication respectively were hypertrophic cardiomyopathy and death, due to the inability of a foetus to adapt. Yet, the study further elaborates on some of the participants being asymptomatic and not as serious. Furthermore, the study further explains the suggested pathophysiology being related to how the foetus may get hypoxemic due to a noted increase in the foetus' oxidative metabolism. ${ }^{9}$

In an article published in the Iranian Journal of Pediatrics "Cardiac Malformations in Fetuses of Gestational and Pre-Gestational Diabetes" ${ }^{\prime 10}$ a case control study was conducted to find the relationship between the two variables. Moreover, the population size included 170 cases and 85 controls. Amongst the diabetic mothers, $8.8 \%$ of the cases reported to have abnormal cardiac findings and only $1.17 \%$ (1 control) of the controls reported to have a cardiac malformation. Furthermore, the study suggested that out of the 180 diabetic mothers, pregestational diabetes showed a higher risk of developing a congenital heart disease compared to gestational diabetes. The writers then suggested that diabetic mothers should get their foetuses screened to prevent extrauterine complications. ${ }^{10}$

A national cohort study was conducted between 1978 and 2011 where the number of cases analysed were over 2 million. The population was designed to analyse only pregestational diabetic mothers. The file records were collected from the National Patient Register and the rest of the information about those patients were taken from pharmacies. The mentioned national study conducted suggested that there is an association between pregestational maternal diabetes mellitus and congenital heart diseases. The study reported that in every 10,000 live births, 318 infants of diabetic mothers would develop congenital heart disease. ${ }^{11}$ On the other hand, a study conducted in Joslin Clinic in Boston reported otherwise. "Congenital heart disease in infants of diabetic mother" 12 an article that was published in The Journal of Pediatrics reviewed and analysed 470 cases and only $4 \%$ of the diabetic population had congenital heart diseases. They concluded that there was no association. ${ }^{12}$

\section{Implications for public health practitioners, clinicians and educators}

After conducting the research, we concluded that there was an association between maternal diabetes and its effects on the development of foetal hearts, therefore, policies in favour of foetal echocardiograph screening must be proposed in maternal clinics and hospitals. Moreover, it is vital that foetuses get screened before their mothers go into labour to take required measures to prevent fatal complications. A foetus with a congenital malformation may either receive drugs as soon as the anomaly is detected, or the mother must be counselled and prepared to have her child go through a possible surgery or procedure to reverse the malformation. Such measures should be taken to physically prepare a foetus for the extra uterine life. Furthermore, it is necessary that mothers get educated on diabetes and its effects on their children and should know about the importance of diet, medications and how to manage and control diabetes. 


\section{Strengths, limitations \& generalisability}

This retrospective cohort study was designed to eliminate all possible biases, including selection bias. Furthermore, the patients selected had no history of other comorbidities and their files had no history of congenital diseases reported. Also, the mothers selected were non- smokers and had no history of drinking alcohol. As for the limitations, although they conducted study is internally valid, strictly to Al Jalila Children's Hospital; it is not yet externally valid. We are unable to compare our data to other hospitals, clinics or institutions. Furthermore, a confounding variable may be the mothers' ages. This study was not age- specific; the mothers were randomly selected if they fulfilled the criteria of having a reported history of diabetes. Even though the cases selected did not include smokers or alcohol drinkers, smoking and alcohol may be additional confounding variables. Smoking and alcohol may not be reported to healthcare professionals in the United Arab of Emirates, especially by females, because the majority find reporting such behaviours a taboo..$^{13}$ Also, as for the cases' management, those recorded included: diet, Glucophage, insulin, or a polytherapy. Although every case had their therapies and management recorded, we do know if the mothers adhered strictly to their management or therapy plan. Moreover, remembering information whilst taking history from the mother such as: history of congenital heart diseases in the family, may introduce recall bias.

\section{Areas for future research}

As this study was a retrospective cohort study that looked at only diabetic mothers, a case control study would be useful to compare control groups (non-diabetic mothers) to cases (diabetic mothers). Comparing cases to controls should give a difference between normal and abnormal. Al Jalila Children's Hospital is a tertiary hospital, which offers subspecialized health services for cases that require more than usual medical attention. Therefore, collecting and analysing data from a secondary hospital may provide more broad information about patients' statuses. Finally, detailed histories must be obtained from the population selected to rule out any confounding variables such as: sociodemographic variables, unclear family history and detailed diet and treatment logs.

\section{Conclusion}

This retrospective cohort study aimed to describe the relationship between maternal diabetes and infants of diabetic mothers, specifically their heart development. After analysing 45 cases from Al Jalila Children's Hospital in a timeframe from 2015 to 2017, the results concluded that there was an association. The possibility of reporting congenital heart diseases in infants of diabetic mothers was high. Furthermore, the results had shown that mothers with diabetes type 1 had a higher risk of carrying foetuses with congenital heart diseases.

\section{Acknowledgments}

My sincere appreciation goes to my very helpful, generous, supportive and knowledgeable supervisor Dr. Mahmoud Al Souf from Al Jalila Children's Hospital and my very helpful, supportive and intelligent Professors of Epidemiology and Research: Dr. Aida Azar, Dr. Tom Loney and Professor Amar Hassan. Thank you for making this possible.

\section{Conflicts of interest}

Authors declare that there is no conflict of interest.

\section{References}

1. United Arab Emirates. International Diabetes Federation-Home.

2. Forbes JM, Cooper ME. Mechanisms of diabetic complications. Physiol Rev. 2013;93(1):137-188.

3. Balsells M, García-Patterson A, Gich I, Major congenital malformations in women with gestational diabetes mellitus: a systematic review and metaanalysis. Diabetes Metab Res Rev. 2012;28:252-257.

4. Becerra JE, Khoury MJ, Cordero JF, et al. Diabetes mellitus during pregnancy and the risks for specific birth defects: a populationbased casecontrol study. Pediatrics. 1990;85(1):1-9.

5. Healthwise Staff. Congenital Heart Defect Types. MyHealth. Alberta. ca Government of Alberta Personal Health Portal. 2017.

6. Webb GD, Smallhorn JF, Therrien J, et al. Congenital heart disease. In: Mann DL, Zipes DP, Libby P, Bonow RO, Braunwald E, editors. Braunwald's Heart Disease: A Textbook of Cardiovascular Medicine. 10th ed. Philadelphia, PA: Elsevier Saunders; 2015. Chap 62.

7. Ministeri M, Alonso-Gonzalez R, Swan L, et al. Common long-term complications of adult congenital heart disease: avoid falling in a H.E.A.P. Expert Rev Cardiovasc Ther. 2016;14(4):445-462.

8. Narchi H, Kulaylat N. Heart disease in infants of diabetic mothers. Images Paediatr Cardiol. 2018;2(2):17-23.

9. Hornberger LK. Maternal diabetes and the fetal heart. Heart. 2006;92(8):1019-1021.

10. Tabib A, Shirzad N, Sheikhbahaei S, et al. Cardiac Malformations in Fetuses of Gestational and Pre Gestational Diabetic Mothers. Iran $J$ Pediatr. 2013;23(6):664-668.

11. Oyen N, Diaz LJ, Mathiesen ER, et al. Prepregnancy Diabetes and Offspring Risk of Congenital Heart Disease A Nationwide Cohort Study. Circulation. 2016;133(23):2243-2253.

12. Rowland TW, Hubbell JP, Nadas AS. Congenital heart disease in infants of diabetic mothers. The Journal of Pediatrics. 1973;83(5):815-820.

13. Al-Houqani M, Leinberger-Jabari A, Al Naeemi A, et al. Patterns of tobacco use in the United Arab Emirates Healthy Future (UAEHFS) pilot study. PLoS One. 2018;13(5):e0198119. 